\title{
STRUCTURE-ACTIVITY RELATIONSHIPS OF VIRGINIAE \\ BUTANOLIDE C, AN INDUCER OF VIRGINIAMYCIN PRODUCTION IN STREPTOMYCES VIRGINIAE
}

\author{
TakuYa Nihira, Yoshihiro Shimizu, Hyun Soo Kim and Yasuhiro Yamada* \\ Department of Fermentation Technology, Faculty of Engineering, Osaka University, \\ 2-1 Yamada-oka, Suita-shi, Osaka 565, Japan
}

(Received for publication June 6, 1988)

Virginiae butanolide C, [2-(1'-hydroxyhexyl)-3-(hydroxymethyl)butanolide (3)], is one of the inducers of virginiamycin production in Streptomyces virginiae. Various racemic analogues were synthesized, and their effectiveness in virginiamycin induction was studied. Among analogues having a series of C-2 side chains, those with 1'-hydroxyheptyl or 1'-hydroxyoctyl moiety were most effective with a minimum effective concentration of $0.8 \mathrm{ng} / \mathrm{ml}$. At the same length of C-2 side chain, a 2,3-cis analogue was 10-fold more active than a 2,3trans analogue, and the 2,3-trans analogue was 10 -fold more active than an analogue having a 1'-ketoalkyl moiety at C-2 (A-factor type analogue). Methoxylation or deletion of either one of the two hydroxy groups in virginiae butanolide $C$ analogues caused a 100 to 1,000-fold decrease in activity, thus indicating the importance of the two hydroxy groups in virginiamycin induction.

Actinomycetes are Gram-positive bacteria characterized by their versatile ability to produce various secondary metabolites, such as antibiotics, and therefore, are one of the most important groups of industrial microorganism. One of the most interesting features of these microorganisms is their

Fig. 1. Structures of signal molecules isolated from Streptomyces.

A-factor from S. griseus.<smiles>CC(C)CCCCC(=O)C1C(=O)OC[C@H]1CO</smiles>

1

Factors from $S$. bikiniensis and S. cyaneofuscatus.<smiles>CC(C)CCC[C@@H](O)[C@H]1C(=O)OC[C@H]1CO</smiles>

3<smiles>CC(C)CCCC[C@H](O)[C@H]1C(=O)OC[C@H]1CO</smiles>

4

Factor I from S. viridochromogenes.<smiles>CC(C)CCCC(O)[C@H]1C(=O)OC[C@H]1CO</smiles>

2

Virginiae butanolides from S. virginiae.<smiles>CC(C)CCCC(O)[C@H]1C(=O)OC[C@H]1CO</smiles>

VB-A (3)<smiles>CCC(C)CCC(O)[C@H]1C(=O)OC[C@H]1CO</smiles>

VB-B (6)<smiles>CC(C)CCCCC[C@H](O)[C@H]1C(=O)OC[C@H]1CO</smiles>

5<smiles>CCCCC[C@@H](O)[C@H]1C(=O)OC[C@H]1CO</smiles>

VB-C (7) 
production of endogeneous and exogeneous signal molecules. These have been called "autoregulators"1,2), and the best studied among them is A-factor (1) from Streptomyces griseus, which is essential for the production of streptomycin and the formation of aerial mycelia ${ }^{3)}$ in some antibiotic- and aerial mycelia-minus mutants. Besides A-factor, additional signal molecules have been isolated and their structures elucidated: Anthracycline-inducing factors (2 5) from Streptomyces viridochromogenes ${ }^{4)}$, Streptomyces bikiniensis and Streptomyces cyaneofuscatus ${ }^{5)}$, B-factor which induces rifamycin production in Nocardia sp. ${ }^{6,7)}, \mathrm{C}$ factor which induces conidia formation in S. griseus ${ }^{8)}$, pamamycin which stimulates aerial mycelia formation in Streptomyces alboniger ${ }^{9)}$, and sporulation pigment from Streptomyces venezuelae ${ }^{10}$.

Recently, we have isolated similar signal molecules named virginiae butanolide A (3), B (6) and C (7) (VB-A, B and C, Fig. 1) from Streptomyces virginiae ${ }^{11}$, which are effective at $1 \mathrm{ng} / \mathrm{ml}$ in initiating virginiamycin production. VBs are 2,3-disubstituted butanolides, and thus, share a common structure with those of A-factor and anthracycline-inducing factors (Fig. 1). This paper deals with structure-activity relationships of various VB-C analogues.

\section{Materials and Methods}

\section{Strain and Medium}

S. virginiae of YANAGIMOTO and TERUI ${ }^{12,13)}$ was used for testing the inducing activity of VB-C analogues. Bacillus subtilis PCI 219 was used to determine the resultant virginiamycin production.

The medium for $S$. virginiae contained Bacto-casitone (Difco) $7.5 \mathrm{~g}$, yeast extract (Oriental Yeast Co., Ltd., Tokyo) $7.5 \mathrm{~g}$, glycerol $15 \mathrm{~g}$ and $\mathrm{NaCl} 2.5 \mathrm{~g}$ per liter, $\mathrm{pH} 6.5$. The medium for $B$. subtilis PCI 219 contained Polypeptone $5 \mathrm{~g}$, meat extract $3 \mathrm{~g}$ and agar $15 \mathrm{~g}$ per liter, $\mathrm{pH}$ 6.5.

Assay of Virginiae Butanolide Activity

Activities of various VB-C analogues and other compounds were assayed in liquid cultures of $S$. virginiae by measuring the production of virginiamycin essentially as described before ${ }^{11}$ except that during the assay culture, $1 \mathrm{ml}$ of an 8-hour cell suspension was added to $1 \mathrm{ml}$ of fresh medium containing appropriately diluted samples in $16.5 \mathrm{~mm}$ test tubes.

\section{Analytical Methods}

IR spectra were determined as films on a Hitachi model 215 grating IR spectrophotometer. NMR spectra at 60,100 and $270 \mathrm{MHz}$ were recorded on a Hitachi model R-24B, a Jeol model PS 100 and a Jeol model JNM-GSX 270 spectrophotometer, respectively. NMR spectra were taken at $100 \mathrm{MHz}$ in $\mathrm{CDCl}_{3}$ unless otherwise described. Chemical shifts were expressed downfield from TMS as an internal standard. Mass spectra were obtained with a Hitachi RMU-6E mass spectrometer.

Synthesis of 3-(Hydroxymethyl)butanolide (16) and 3-(Trimethylsilyloxymethyl)butanolide (17)

3-(Hydroxymethyl)butanolide (16) was synthesized by reduction of diethyl formylsuccinate with $\mathrm{NaBH}_{4}$, and 3-(trimethylsilyloxymethyl)butanolide (17) by trimethylsilylation of 16 as previously described $^{11}$.

\section{Synthesis of 3-(Methoxymethyl)butanolide (18)}

To a solution of 3-(hydroxymethyl)butanolide (16) (3 g) and 1,10-phenanthroline in $20 \mathrm{ml}$ of dry benzene, $n$-butyl lithium (1.1 equiv, hexane solution) was added dropwise at room temperature under a nitrogen atmosphere with stirring. After standing for 1 hour, methyl iodide (6 equiv) was added and incubated overnight. The reaction was stopped by the addition of $\mathrm{MeOH}$, the mixture was poured into $100 \mathrm{ml}$ of $1 \mathrm{~N} \mathrm{HCl}$ and extracted with dichloromethane. The dichloromethane layer was dried over anhydrous sodium sulfate, concentrated to dryness, and the resulting oil ( $4.43 \mathrm{~g}$ ) was purified on a silica gel $(70 \mathrm{~g})$ column developed with $n$-hexane - EtOAc $(6: 4)$. Yield of 18, $0.23 \mathrm{~g}:{ }^{1} \mathrm{H}$ NMR 
$\left(100 \mathrm{MHz}, \mathrm{CDCl}_{3}\right) \delta 4.48 \sim 4.04\left(2 \mathrm{H}, \mathrm{m}, 4-\mathrm{H}_{2}\right), 3.48 \sim 3.32\left(5 \mathrm{H}, \mathrm{m}, \mathrm{CH}_{2} \mathrm{OCH}_{3}\right), 2.98 \sim 2.68(1 \mathrm{H}, \mathrm{m}$, 3-H), $2.68 \sim 2.36\left(2 \mathrm{H}, \mathrm{m}, 2-\mathrm{H}_{2}\right)$; IR (film) $\mathrm{cm}^{-1} 3500,2900,1780$; electron impact mass spectra (EIMS) $m / z 130(\mathrm{M}), 100\left(\mathrm{M}-\mathrm{OCH}_{3}\right)$.

\section{Synthesis of 3-Methylbutanolide (19)}

1) n-Octyl 3-(Carboxy)-3-butenoate (20): Itaconic anhydride (21, $25 \mathrm{~g}$ ) was mixed with $n$ octanol $\left(38.6 \mathrm{ml}, 1.1\right.$ equiv), and the mixture was stirred at $110^{\circ} \mathrm{C}$. After a 6-hour reaction, $100 \mathrm{ml}$ of water was added and stirred for a further 30 minutes. The reaction mixture was extracted with hexane $(300 \mathrm{ml})$, and the extract was washed with water $(150 \mathrm{ml}$, twice), dried over anhydrous sodium sulfate, and evaporated. The resulting oil $(51.6 \mathrm{~g})$ was distilled in vacuum to remove the remaining $n$-octanol and $45.2 \mathrm{~g}$ of 20 was obtained: ${ }^{1} \mathrm{H}$ NMR $\left(60 \mathrm{MHz}, \mathrm{CDCl}_{3}\right) \delta 6.37(1 \mathrm{H}, \mathrm{s}), 5.74(1 \mathrm{H}, \mathrm{s}), 4.04$ $(2 \mathrm{H}, \mathrm{t}), 1.24(14 \mathrm{H}, \mathrm{br}), 0.82(3 \mathrm{H}, \mathrm{t})$; IR (film) $\mathrm{cm}^{-1} 2916,2850,1724,1684,1638$.

2) $n$-Octyl 3-(Carboxy)butyrate (21): $20(20 \mathrm{~g})$ dissolved in $200 \mathrm{ml}$ of EtOAc was catalytically hydrogenated with $5 \% \mathrm{Pd}-\mathrm{C}(1.5 \mathrm{~g})$ at room temperature for 6 hours. The catalyst was removed by suction filtration, and the filtrate was concentrated to dryness yielding $20.2 \mathrm{~g}$ of $21:{ }^{1} \mathrm{H}$ NMR (100 $\left.\mathrm{MHz}, \mathrm{CDCl}_{3}\right) \delta 4.04(2 \mathrm{H}, \mathrm{t}), 2.61(1 \mathrm{H}, \mathrm{q}), 1.4(14 \mathrm{H}, \mathrm{b}), 1.2(3 \mathrm{H}, \mathrm{d}), 0.88(3 \mathrm{H}, \mathrm{t}) ; \mathrm{IR}$ (film) $\mathrm{cm}^{-1} 3200$, $2916,2850,1740,1712$.

3) 3-Methylbutanolide (22): To a solution of $21(5 \mathrm{~g})$ in $50 \mathrm{ml}$ of dry THF, borane-methyl sulfide complex ( $2 \mathrm{M}$ solution in THF, $20.6 \mathrm{ml}$ ) was added dropwise with stirring at $-5^{\circ} \mathrm{C}$ for 1 hour. The reaction was continued for a further 6 hours. $\mathrm{MeOH}(25 \mathrm{ml})$ was added dropwise, and the reaction mixture was evaporated. The residue was again dissolved in $\mathrm{MeOH}$ and evaporated, yielding $5 \mathrm{~g}$ of $n$-octyl 3-(hydroxymethyl)butyrate (23): ${ }^{1} \mathrm{H}$ NMR of $23\left(100 \mathrm{MHz}, \mathrm{CDCl}_{3}\right) \delta 4.02(2 \mathrm{H}, \mathrm{t}), 3.45$ $(2 \mathrm{H}, \mathrm{d}), 2.25(1 \mathrm{H}, \mathrm{q}), 1.3(14 \mathrm{H}, \mathrm{br}), 1.0(3 \mathrm{H}), 0.88(3 \mathrm{H}, \mathrm{t})$; IR (film) $\mathrm{cm}^{-1} 3400,2920,2850,1740$.

$23(5 \mathrm{~g})$ was hydrolyzed in a mixture of $\mathrm{EtOH}(50 \mathrm{ml}), \mathrm{KOH}(2$ equiv) and water $(20 \mathrm{ml})$ by refluxing at $110^{\circ} \mathrm{C}$ for 1 hour. After removing EtOH by evaporation, water $(50 \mathrm{ml})$ was added, and the reaction mixture was extracted by hexane to remove $n$-octanol. After acidification with conc $\mathrm{HCl}$, the water layer was extracted with dichloromethane, and the extract was dried and evaporated yielding $1.67 \mathrm{~g}$ of crude oil. The oil was purified on a silica gel $(30 \mathrm{~g})$ column eluted with dichloromethane. Yield of $22 ; 1 \mathrm{~g}:{ }^{1} \mathrm{H}$ NMR $\left(100 \mathrm{MHz}, \mathrm{CDCl}_{3}\right) \delta 4.5 \sim 4.28(1 \mathrm{H}, \mathrm{m}), 3.98 \sim 3.78(1 \mathrm{H}, \mathrm{m})$, $2.78 \sim 2.58(2 \mathrm{H}, \mathrm{d}), 2.28 \sim 1.88(1 \mathrm{H}, \mathrm{m}), 1.21 \sim 1.04(3 \mathrm{H}, \mathrm{d})$; IR (film) $\mathrm{cm}^{-1} 2970,2905,1786$.

\section{Synthesis of Racemic Virginiae Butanolide C Analogues (8 15)}

A-Factor type analogues (8) with various alkyl side chains at $\mathrm{C}-2$ were synthesized essentially as described before ${ }^{11)}$ by reacting 3-(trimethylsilyloxymethyl)butanolide (17) with a suitable alkyl chloride. A-Factor type analogue (8) thus obtained was reduced with $\mathrm{NaBH}_{4}$ yielding a mixture of cis (10) and trans (9) isomers, and these isomers were separated by reverse phase HPLC. For 6-deoxy analogues (14 and 15), heptyl iodide was used instead of heptanoyl chloride. For the syntheses of 3-methoxymethyl analogues (11a, 12a and 13a), 3-methyl analogues (11b, 12b and 13b) and 3-nor analogues (11c and 12c), 3-(methoxymethyl)butanolide (18), 3-methylbutanolide (19) and butanolide, respectively, were used instead of 3-(trimethylsilyloxymethyl)butanolide (17).

8a: MS $m / z 158\left(\mathrm{M}, \mathrm{C}_{7} \mathrm{H}_{10} \mathrm{O}_{4}\right)$; IR (film) $\mathrm{cm}^{-1} 3450,2950,1760,1680,1470,1430 ;{ }^{1} \mathrm{H}$ NMR $(60 \mathrm{MHz}) \delta 1.7$ and $2.5\left(3 \mathrm{H}, \mathrm{s}, \mathrm{CH}_{3}\right.$, enol and keto), $3.2 \sim 3.3(1 \mathrm{H}, \mathrm{m}), 3.6 \sim 3.8(2 \mathrm{H}, \mathrm{m}), 4.0 \sim 4.5(3 \mathrm{H}$, $\mathrm{m})$; yield $25 \%$.

8b: MS $m / z 172(\mathrm{M})$; IR (film) $\mathrm{cm}^{-1} 3430,2950,1760,1720,1620,1460 ;{ }^{1} \mathrm{H}$ NMR $\delta 1.0 \sim 1.2$ $(3 \mathrm{H}, \mathrm{t}), 2.6 \sim 3.3(3 \mathrm{H}, \mathrm{m}), 3.6 \sim 3.8(2 \mathrm{H}, \mathrm{m}), 4.0 \sim 4.6(3 \mathrm{H}, \mathrm{m})$; yield $22 \%$.

8c: MS $m / z 186(\mathrm{M})$; IR (film) $\mathrm{cm}^{-1} 3450,2960,1770,1720,1640,1470 ;{ }^{1} \mathrm{H}$ NMR $\delta 0.80 \sim 1.00$ $(3 \mathrm{H}, \mathrm{t}), 1.4 \sim 1.8(2 \mathrm{H}, \mathrm{m}), 2.3 \sim 3.3(3 \mathrm{H}, \mathrm{m}), 3.5 \sim 3.8(2 \mathrm{H}, \mathrm{m}), 4.0 \sim 4.6(3 \mathrm{H}, \mathrm{m}) ;$ yield $34 \%$.

8d: $\quad$ MS $m / z 200(\mathrm{M}), 182\left(\mathrm{M}-\mathrm{H}_{2} \mathrm{O}\right)$; IR (film) $\mathrm{cm}^{-1} 3450,2950,1760,1720,1640,1470 ;{ }^{1} \mathrm{H}$ NMR $\delta 0.8 \sim 1.0(3 \mathrm{H}, \mathrm{t}), 1.2 \sim 1.8(6 \mathrm{H}, \mathrm{m}), 2.4 \sim 3.3(3 \mathrm{H}, \mathrm{m}), 3.6 \sim 3.8(2 \mathrm{H}, \mathrm{m}), 4.0 \sim 4.6(3 \mathrm{H}, \mathrm{m})$; yield $26 \%$.

8e: IR (film) $\mathrm{cm}^{-1} 3450,1770,1720 ;{ }^{1} \mathrm{H}$ NMR $\delta 0.9(3 \mathrm{H}, \mathrm{t}), 1.0 \sim 2.0(6 \mathrm{H}, \mathrm{m}), 2.5 \sim 3.4(3 \mathrm{H}, \mathrm{m})$, $3.5 \sim 3.8(2 \mathrm{H}, \mathrm{m}), 3.7 \sim 4.6(3 \mathrm{H}, \mathrm{m})$; yield $27 \%$.

8f: MS $m / z 228(\mathrm{M}), 210\left(\mathrm{M}-\mathrm{H}_{2} \mathrm{O}\right)$; IR (film) $\mathrm{cm}^{-1} 3450,2940,1760,1720,1640 ;{ }^{1} \mathrm{H}$ NMR $\delta$ 
$0.8 \sim 1.0(3 \mathrm{H}, \mathrm{t}), 1.1 \sim 1.8(8 \mathrm{H}, \mathrm{br}), 2.6 \sim 3.3(3 \mathrm{H}, \mathrm{m}), 3.6 \sim 3.8(2 \mathrm{H}, \mathrm{m}), 4.0 \sim 4.6(3 \mathrm{H}, \mathrm{m})$; yield $32 \%$.

8g: MS $m / z 242(\mathrm{M}), 224\left(\mathrm{M}-\mathrm{H}_{2} \mathrm{O}\right)$; IR (film) $\mathrm{cm}^{-1} 3450,2940,1770,1720,1620 ;{ }^{1} \mathrm{H}$ NMR $\delta 0.8 \sim 1.0(3 \mathrm{H}, \mathrm{t}), 1.2 \sim 1.8(10 \mathrm{H}, \mathrm{br}), 2.4 \sim 3.3(3 \mathrm{H}, \mathrm{m}), 3.5 \sim 3.8(2 \mathrm{H}, \mathrm{m}), 4.0 \sim 4.5(3 \mathrm{H}, \mathrm{m}) ;$ yield $25 \%$.

8h: MS $m / z 256(\mathrm{M}), 238\left(\mathrm{M}-\mathrm{H}_{2} \mathrm{O}\right)$; IR (film) $\mathrm{cm}^{-1} 3450,2950,2850,1760,1720 ;{ }^{1} \mathrm{H}$ NMR $\delta 0.8 \sim 1.0(3 \mathrm{H}, \mathrm{t}), 1.2 \sim 1.8(12 \mathrm{H}), 2.5 \sim 3.4(3 \mathrm{H}, \mathrm{m}), 3.6 \sim 3.8(2 \mathrm{H}, \mathrm{m}), 4.0 \sim 4.5(3 \mathrm{H}, \mathrm{m}) ;$ yield $24 \%$.

8i: $\quad$ MS $m / z 270(\mathrm{M}), 252\left(\mathrm{M}-\mathrm{H}_{2} \mathrm{O}\right)$; IR (film) $\mathrm{cm}^{-1} 3450,2950,2870,1780,1730 ;{ }^{1} \mathrm{H}$ NMR $\delta 0.8 \sim 1.0(3 \mathrm{H}, \mathrm{t}), 1.2 \sim 1.8(14 \mathrm{H}, \mathrm{br}), 2.5 \sim 3.4(3 \mathrm{H}, \mathrm{m}), 3.6 \sim 3.8(2 \mathrm{H}, \mathrm{m}), 4.0 \sim 4.5(3 \mathrm{H}, \mathrm{m}) ;$ yield $34 \%$.

9a: MS $m / z 160(\mathrm{M}), 142\left(\mathrm{M}-\mathrm{H}_{2} \mathrm{O}\right)$; IR (film) $\mathrm{cm}^{-1} 3350,2960,2900,1760$; ${ }^{1} \mathrm{H}$ NMR $\delta 1.2 \sim 1.4$ $(2 \mathrm{H}, \mathrm{d}), 2.4 \sim 3.1\left(2 \mathrm{H}, \mathrm{m}, J_{2,3}=8.8 \mathrm{~Hz}\right), 3.3 \sim 3.5(1 \mathrm{H}, \mathrm{br}), 3.6 \sim 3.8(2 \mathrm{H}, \mathrm{m}), 3.9 \sim 4.5(3 \mathrm{H}, \mathrm{m})$; yield $5 \%$.

9b: $\quad$ MS $m / z 174(\mathrm{M}), 156\left(\mathrm{M}-\mathrm{H}_{2} \mathrm{O}\right)$; IR (film) $\mathrm{cm}^{-1} 3400,2950,2880,1760 ;{ }^{1} \mathrm{H}$ NMR $\delta 0.9 \sim$ $1.1(3 \mathrm{H}, \mathrm{t}), 1.5 \sim 1.9(2 \mathrm{H}, \mathrm{m}), 2.6 \sim 2.9\left(2 \mathrm{H}, \mathrm{m}, J_{2,3}=8.8 \mathrm{~Hz}\right), 3.1 \sim 3.3(1 \mathrm{H}, \mathrm{br}), 3.6 \sim 3.8(2 \mathrm{H}, \mathrm{m})$, $3.9 \sim 4.5(3 \mathrm{H}, \mathrm{m})$; yield $12 \%$.

9c: $\operatorname{MS~} m / z 188(\mathrm{M}), 170\left(\mathrm{M}-\mathrm{H}_{2} \mathrm{O}\right)$; IR (film) $\mathrm{cm}^{-1} 3400,2950,2870,1760 ;{ }^{1} \mathrm{H}$ NMR $\delta 0.8 \sim$ $1.1(3 \mathrm{H}, \mathrm{t}), 1.4 \sim 1.8(4 \mathrm{H}, \mathrm{m}), 2.5 \sim 2.9\left(2 \mathrm{H}, \mathrm{m}, J_{2,3}=8.8 \mathrm{~Hz}\right), 3.1 \sim 3.5(2 \mathrm{H}, \mathrm{br}), 3.6 \sim 3.8(2 \mathrm{H}, \mathrm{m})$, $3.9 \sim 4.5(3 \mathrm{H}, \mathrm{m})$; yield $15 \%$.

9d: $\mathrm{MS} m / z 202(\mathrm{M}), 184\left(\mathrm{M}-\mathrm{H}_{2} \mathrm{O}\right)$; IR (film) $\mathrm{cm}^{-1} 3400,2950,2850,1760 ;{ }^{1} \mathrm{H}$ NMR $\delta 0.8 \sim$ $1.1(3 \mathrm{H}, \mathrm{t}), 1.2 \sim 2.0(6 \mathrm{H}, \mathrm{m}), 2.5 \sim 2.9\left(2 \mathrm{H}, \mathrm{m}, J_{2,3}=8.8 \mathrm{~Hz}\right), 3.0 \sim 3.4(2 \mathrm{H}, \mathrm{br}), 3.6 \sim 3.8(2 \mathrm{H}, \mathrm{m})$, $3.9 \sim 4.5(3 \mathrm{H}, \mathrm{m})$; yield $10 \%$.

9e: As described before ${ }^{11}$.

9f: MS $m / z 230(\mathrm{M}), 212\left(\mathrm{M}-\mathrm{H}_{2} \mathrm{O}\right)$; IR (film) $\mathrm{cm}^{-1} 3400,2930,2850,1760$; ${ }^{1} \mathrm{H}$ NMR $\delta 0.8 \sim$ $1.0(3 \mathrm{H}, \mathrm{t}), 1.2 \sim 1.8(10 \mathrm{H}, \mathrm{m}), 2.5 \sim 2.9\left(2 \mathrm{H}, \mathrm{m}, J_{2,3}=8.8 \mathrm{~Hz}\right), 2.9 \sim 3.4(2 \mathrm{H}, \mathrm{br}), 3.6 \sim 3.8(2 \mathrm{H}, \mathrm{m})$, $3.9 \sim 4.5(3 \mathrm{H}, \mathrm{m})$; yield $6 \%$.

9g: MS $m / z 244(\mathrm{M}), 226\left(\mathrm{M}-\mathrm{H}_{2} \mathrm{O}\right)$; IR (film) $\mathrm{cm}^{-1} 3400,2930,2850,1760 ;{ }^{1} \mathrm{H}$ NMR $\delta 0.8 \sim$ $1.0(3 \mathrm{H}, \mathrm{t}), 1.2 \sim 1.8(12 \mathrm{H}, \mathrm{m}), 2.5 \sim 2.9\left(2 \mathrm{H}, \mathrm{m}, J_{2,3}=8.8 \mathrm{~Hz}\right), 3.6 \sim 3.8(2 \mathrm{H}, \mathrm{m}), 3.9 \sim 4.5(3 \mathrm{H}, \mathrm{m})$; yield $13 \%$.

9h: $\mathrm{MS} m / z 258(\mathrm{M}), 240\left(\mathrm{M}-\mathrm{H}_{2} \mathrm{O}\right)$; IR (film) $\mathrm{cm}^{-1} 3400,2950,2850,1760 ;{ }^{1} \mathrm{H}$ NMR $\delta 0.8 \sim$ $1.0(3 \mathrm{H}, \mathrm{t}), 1.2 \sim 1.8(14 \mathrm{H}, \mathrm{m}), 2.5 \sim 2.9\left(2 \mathrm{H}, \mathrm{m}, J_{2,3}=8.8 \mathrm{~Hz}\right), 3.5 \sim 3.8(2 \mathrm{H}, \mathrm{m}), 3.9 \sim 4.5(3 \mathrm{H}, \mathrm{m})$; yield $8 \%$.

9i: $\quad$ MS $m / z 272$ (M), $254\left(\mathrm{M}-\mathrm{H}_{2} \mathrm{O}\right)$; IR (film) $\mathrm{cm}^{-1} 3400,2950,2860,1780 ;{ }^{1} \mathrm{H}$ NMR $\delta 0.8 \sim$ $1.0(3 \mathrm{H}, \mathrm{t}), 1.2 \sim 1.8(16 \mathrm{H}, \mathrm{m}), 2.5 \sim 2.9\left(2 \mathrm{H}, \mathrm{m}, J_{2,3}=8.8 \mathrm{~Hz}\right), 3.4 \sim 3.6(2 \mathrm{H}, \mathrm{br}), 3.6 \sim 3.8(2 \mathrm{H}, \mathrm{m})$, $3.9 \sim 4.5(3 \mathrm{H}, \mathrm{m})$; yield $6 \%$.

9j: MS $m / z 200(\mathrm{M}), 182\left(\mathrm{M}-\mathrm{H}_{2} \mathrm{O}\right)$; IR (film) $\mathrm{cm}^{-1} 3400,2920,2850,1760 ;{ }^{1} \mathrm{H}$ NMR $\delta 1.6 \sim$ $1.8(2 \mathrm{H}, \mathrm{m}), 2.0 \sim 2.4(2 \mathrm{H}, \mathrm{m}), 2.5 \sim 2.9\left(2 \mathrm{H}, \mathrm{m}, J_{2,3}=8.8 \mathrm{~Hz}\right), 3.4 \sim 3.8(2 \mathrm{H}, \mathrm{m}), 3.9 \sim 4.2(2 \mathrm{H}, \mathrm{m})$, $4.3 \sim 4.5(1 \mathrm{H}, \mathrm{m}), 4.9 \sim 5.2(2 \mathrm{H}, \mathrm{m}), 5.6 \sim 6.0(1 \mathrm{H}, \mathrm{m})$; yield $1 \%$.

9k: IR (film) $\mathrm{cm}^{-1} 3370,3070,2930,2850,1760,1640 ;{ }^{1} \mathrm{H}$ NMR $\delta 1.2 \sim 1.8(6 \mathrm{H}, \mathrm{m}), 2.0 \sim 2.3$ $(2 \mathrm{H}, \mathrm{m}), 2.6 \sim 3.0\left(2 \mathrm{H}, \mathrm{m}, J_{2,3}=8.8 \mathrm{~Hz}\right), 3.4 \sim 3.9(4 \mathrm{H}, \mathrm{m}), 3.9 \sim 4.2(2 \mathrm{H}, \mathrm{m}), 4.3 \sim 4.6(1 \mathrm{H}, \mathrm{m}), 4.9 \sim$ $5.2(2 \mathrm{H}, \mathrm{m}), 5.6 \sim 6.0(1 \mathrm{H}, \mathrm{m})$.

10a: MS $m / z 142\left(\mathrm{M}-\mathrm{H}_{2} \mathrm{O}\right)$; IR (film) $\mathrm{cm}^{-1} 3400,3000,2880,1780,1680 ;{ }^{1} \mathrm{H}$ NMR $\delta 1.4(3 \mathrm{H}$, d, $J=7.2 \mathrm{~Hz}), 2.2 \sim 2.9\left(2 \mathrm{H}, \mathrm{m}, J_{2,3}=7.1 \mathrm{~Hz}\right), 3.6 \sim 3.8(2 \mathrm{H}, \mathrm{d}, J=6.5 \mathrm{~Hz}), 3.9 \sim 4.5(3 \mathrm{H}, \mathrm{m})$; yield $2 \%$.

10b: MS $m / z 174(\mathrm{M}), 156\left(\mathrm{M}-\mathrm{H}_{2} \mathrm{O}\right)$; IR (film) $\mathrm{cm}^{-1} 3400,3000,2880,1780,1680 ;{ }^{1} \mathrm{H}$ NMR $\delta 0.9 \sim 1.1(3 \mathrm{H}, \mathrm{t}), 1.6 \sim 1.8(2 \mathrm{H}, \mathrm{m}), 2.2 \sim 2.9\left(2 \mathrm{H}, \mathrm{m}, J_{2,3}=7.1 \mathrm{~Hz}\right), 3.6 \sim 3.8(2 \mathrm{H}, \mathrm{d}, J=6.5 \mathrm{~Hz}), 3.9 \sim$ $4.5(3 \mathrm{H}, \mathrm{m})$; yield $5 \%$.

10c: MS $m / z 188(\mathrm{M}), 170\left(\mathrm{M}-\mathrm{H}_{2} \mathrm{O}\right)$; IR (film) $\mathrm{cm}^{-1} 3400,2950,2850,1750,1650 ;{ }^{1} \mathrm{H}$ NMR $\delta 0.9 \sim 1.1(3 \mathrm{H}, \mathrm{t}), 1.3 \sim 1.8(4 \mathrm{H}, \mathrm{m}), 2.4 \sim 2.9\left(2 \mathrm{H}, \mathrm{m}, J_{2,3}=7.1 \mathrm{~Hz}\right), 3.6 \sim 3.8(2 \mathrm{H}, J=6.2 \mathrm{~Hz}), 4.0 \sim$ $4.5(3 \mathrm{H}, \mathrm{m})$; yield $8 \%$.

10d: MS $m / z 202(\mathrm{M}), 184\left(\mathrm{M}-\mathrm{H}_{2} \mathrm{O}\right)$; IR (film) $\mathrm{cm}^{-1} 3400,2950,2840,1760,1660 ;{ }^{1} \mathrm{H}$ NMR $\delta 0.8 \sim 1.0(3 \mathrm{H}, \mathrm{t}), 1.2 \sim 1.8(6 \mathrm{H}, \mathrm{m}), 2.4 \sim 2.9\left(2 \mathrm{H}, \mathrm{m}, J_{2,3}=7.1 \mathrm{~Hz}\right), 3.6 \sim 3.8(2 \mathrm{H}, \mathrm{d}, J=6.2 \mathrm{~Hz}), 4.0 \sim$ $4.5(3 \mathrm{H}, \mathrm{m})$; yield $4 \%$.

10e: As described before ${ }^{11)}$.

10f: MS $\mathrm{m} / \mathrm{z} 230(\mathrm{M}), 212\left(\mathrm{M}-\mathrm{H}_{2} \mathrm{O}\right)$; IR (film) $\mathrm{cm}^{-1} 3400,2950,2840,1760,1660 ;{ }^{1} \mathrm{H}$ NMR $\delta 0.8 \sim 1.0(3 \mathrm{H}, \mathrm{t}), 1.2 \sim 1.8(10 \mathrm{H}, \mathrm{m}), 2.4 \sim 2.9\left(2 \mathrm{H}, \mathrm{m}, J_{2,3}=7.1 \mathrm{~Hz}\right), 3.6 \sim 3.8(2 \mathrm{H}, \mathrm{d}, J=6.1 \mathrm{~Hz})$, 
$4.0 \sim 4.5(3 \mathrm{H}, \mathrm{m})$; yield $4 \%$.

10g: MS m/z $244(\mathrm{M}), 226\left(\mathrm{M}-\mathrm{H}_{2} \mathrm{O}\right)$; IR (film) $\mathrm{cm}^{-1} 3400,2950,2850,1760,1660 ;{ }^{1} \mathrm{H}$ NMR $\delta 0.8 \sim 1.0(3 \mathrm{H}, \mathrm{t}), 1.2 \sim 1.8(12 \mathrm{H}, \mathrm{m}), 2.4 \sim 2.9\left(2 \mathrm{H}, \mathrm{m}, J_{2,3}=7.1 \mathrm{~Hz}\right), 3.6 \sim 3.8(2 \mathrm{H}, \mathrm{d}, J=6.1 \mathrm{~Hz})$, $4.0 \sim 4.5(3 \mathrm{H}, \mathrm{m})$; yield $6 \%$.

10h: MS $m / z 258(\mathrm{M}), 240\left(\mathrm{M}-\mathrm{H}_{2} \mathrm{O}\right)$; IR (film) $\mathrm{cm}^{-1} 3400,2950,2850,1760$; ${ }^{1} \mathrm{H}$ NMR $\delta 0.8 \sim$ $1.0(3 \mathrm{H}, \mathrm{t}), 1.2 \sim 1.8(14 \mathrm{H}, \mathrm{m}), 2.4 \sim 3.1\left(2 \mathrm{H}, \mathrm{m}, J_{2,3}=7.1 \mathrm{~Hz}\right), 3.6 \sim 3.8(2 \mathrm{H}, \mathrm{d}, J=6.1 \mathrm{~Hz}), 4.0 \sim 4.5$ $(3 \mathrm{H}, \mathrm{m})$; yield $5 \%$.

10i: $\mathrm{MS} m / z 272(\mathrm{M}), 254\left(\mathrm{M}-\mathrm{H}_{2} \mathrm{O}\right)$; IR (film) 3400, 2950, 2850, 1760; ${ }^{1} \mathrm{H}$ NMR $\delta 0.8 \sim 1.0$ $(3 \mathrm{H}, \mathrm{t}), 1.2 \sim 1.8(16 \mathrm{H}, \mathrm{m}), 2.4 \sim 3.0\left(2 \mathrm{H}, \mathrm{m}, J_{2,3}=7.1 \mathrm{~Hz}\right), 3.6 \sim 3.8(2 \mathrm{H}, \mathrm{d}, J=6.1 \mathrm{~Hz}), 4.0 \sim 4.5(3 \mathrm{H}$, $\mathrm{m})$; yield $3 \%$.

10j: MS $m / z 200(\mathrm{M}), 182\left(\mathrm{M}-\mathrm{H}_{2} \mathrm{O}\right)$; IR (film) $\mathrm{cm}^{-1} 3400,2920,2850,1760 ;{ }^{1} \mathrm{H}$ NMR $\delta 1.6 \sim$ $1.9(2 \mathrm{H}, \mathrm{m}), 2.0 \sim 2.4(2 \mathrm{H}, \mathrm{m}), 2.4 \sim 2.9\left(2 \mathrm{H}, \mathrm{m}, J_{2,3}=7.1 \mathrm{~Hz}\right), 3.6 \sim 3.8(2 \mathrm{H}, \mathrm{d}, J=6.1 \mathrm{~Hz}), 4.0 \sim 4.5$ $(3 \mathrm{H}, \mathrm{m}), 4.9 \sim 5.2(2 \mathrm{H}, \mathrm{m}), 5.6 \sim 6.0(1 \mathrm{H}, \mathrm{m})$; yield $1 \%$.

10k: IR (film) $\mathrm{cm}^{-1} 3370,3070,2930,2850,1750,1640 ;{ }^{1} \mathrm{H}$ NMR $\delta 1.2 \sim 1.8(6 \mathrm{H}, \mathrm{m}), 2.0 \sim 2.3$ $(2 \mathrm{H}, \mathrm{m}), 2.4 \sim 2.7\left(1 \mathrm{H}, \mathrm{m}, J_{\mathrm{z}, 3}=6.5 \mathrm{~Hz}\right), 2.7 \sim 3.0(2 \mathrm{H}, \mathrm{m}), 3.0 \sim 3.2(1 \mathrm{H}, \mathrm{m}), 3.6 \sim 3.9(2 \mathrm{H}, \mathrm{m}), 4.0 \sim$ $4.2(2 \mathrm{H}, \mathrm{m}), 4.3 \sim 4.6(1 \mathrm{H}, \mathrm{m}), 4.8 \sim 5.2(2 \mathrm{H}, \mathrm{m}), 5.6 \sim 6.0(1 \mathrm{H}, \mathrm{m})$; yield $3 \%$.

11a: MS $m / z 242(\mathrm{M}), 224\left(\mathrm{M}-\mathrm{H}_{2} \mathrm{O}\right)$; IR (film) $\mathrm{cm}^{-1} 2930,2850,1780,1720 ;{ }^{1} \mathrm{H}$ NMR $\delta 0.8 \sim$ $1.0(3 \mathrm{H}, \mathrm{t}), 1.2 \sim 1.8(8 \mathrm{H}, \mathrm{m}), 2.5 \sim 2.9(2 \mathrm{H}, \mathrm{m}), 3.2 \sim 3.5(5 \mathrm{H}, \mathrm{m}), 3.6 \sim 3.8(1 \mathrm{H}, \mathrm{m}), 4.0 \sim 4.5(2 \mathrm{H}, \mathrm{m})$; yield $37 \%$.

11b: IR (film) $\mathrm{cm}^{-1} 2940,2860,1780,1720 ;{ }^{1} \mathrm{H}$ NMR $\delta 0.8 \sim 1.0(3 \mathrm{H}, \mathrm{t}), 1.0 \sim 1.2(3 \mathrm{H}, \mathrm{d}, J=$ $7.2 \mathrm{~Hz}), 1.2 \sim 1.8(8 \mathrm{H}, \mathrm{m}), 2.4 \sim 3.2(2 \mathrm{H}, \mathrm{m}), 3.2 \sim 3.4(2 \mathrm{H}, \mathrm{m}), 3.7 \sim 3.9(1 \mathrm{H}, \mathrm{m}), 4.3 \sim 4.6(1 \mathrm{H}, \mathrm{m})$; yield $25 \%$.

11c: MS $m / z 198(\mathrm{M})$; IR (film) $\mathrm{cm}^{-1} 2930,2850,1770,1720 ;{ }^{1} \mathrm{H}$ NMR $\delta 0.8 \sim 1.0(3 \mathrm{H}, \mathrm{t}), 1.2 \sim$ $1.8(8 \mathrm{H}, \mathrm{m}), 2.2 \sim 3.0(4 \mathrm{H}, \mathrm{m}), 3.6 \sim 3.9(1 \mathrm{H}, \mathrm{m}), 4.2 \sim 4.4(2 \mathrm{H}, \mathrm{m})$; yield $55 \%$.

12a: IR (film) $\mathrm{cm}^{-1} 3450,2940,2850,1760 ;{ }^{1} \mathrm{H}$ NMR $\delta 0.8 \sim 1.0(3 \mathrm{H}, \mathrm{t}), 1.2 \sim 1.8(10 \mathrm{H}, \mathrm{m})$, $2.4 \sim 2.6\left(1 \mathrm{H}, \mathrm{m}, J_{2,3}=9.0 \mathrm{~Hz}\right), 2.7 \sim 3.1(1 \mathrm{H}, \mathrm{m}), 3.3 \sim 3.5(5 \mathrm{H}, \mathrm{m}), 4.0 \sim 4.5(3 \mathrm{H}, \mathrm{m})$; yield $1 \%$.

12b: IR (film) cm ${ }^{-1} 3400,2940,2850,1760 ;{ }^{1} \mathrm{H}$ NMR $\delta 0.8 \sim 1.0(3 \mathrm{H}, \mathrm{t}), 1.1 \sim 1.2(3 \mathrm{H}, \mathrm{d}, J=$ $7.1 \mathrm{~Hz}), 1.2 \sim 1.8(10 \mathrm{H}, \mathrm{m}), 2.2 \sim 2.4\left(1 \mathrm{H}, \mathrm{m}, J_{2,3}=11.4 \mathrm{~Hz}\right), 2.6 \sim 3.0(1 \mathrm{H}, \mathrm{m}), 3.6 \sim 3.9(1 \mathrm{H}, \mathrm{m}), 4.0 \sim$ $4.2(1 \mathrm{H}, \mathrm{br}), 4.3 \sim 4.5(1 \mathrm{H}, \mathrm{m})$; yield $1 \%$.

12c: IR (film) $\mathrm{cm}^{-1} 3450,2920,2850,1760 ;{ }^{1} \mathrm{H}$ NMR $\delta 0.8 \sim 1.0(3 \mathrm{H}, \mathrm{t}), 1.2 \sim 1.7(10 \mathrm{H}, \mathrm{m})$, $2.1 \sim 3.0(3 \mathrm{H}, \mathrm{m}), 3.6 \sim 4.0(1 \mathrm{H}, \mathrm{br}), 4.0 \sim 4.5(3 \mathrm{H}, \mathrm{m})$; yield $3 \%$.

13a: IR (film) cm $\mathrm{cm}^{-1} 3450,2930,2850,1770 ;{ }^{1} \mathrm{H}$ NMR $\delta 0.8 \sim 1.0(3 \mathrm{H}, \mathrm{t}), 1.2 \sim 1.7(10 \mathrm{H}, \mathrm{m})$, $2.5 \sim 2.9\left(2 \mathrm{H}, \mathrm{m}, J_{2,3}=7.2 \mathrm{~Hz}\right), 3.3 \sim 3.5(5 \mathrm{H}, \mathrm{m}), 3.8 \sim 4.1(1 \mathrm{H}, \mathrm{m}), 4.3 \sim 4.5(1 \mathrm{H}, \mathrm{m})$; yield $3 \%$.

13b: IR (film) 3450, 2940, 2850, 1760; ${ }^{1} \mathrm{H}$ NMR $\delta 0.8 \sim 1.0(3 \mathrm{H}, \mathrm{t}), 1.1 \sim 1.2(3 \mathrm{H}, \mathrm{d}, J=7.1 \mathrm{~Hz})$, $1.2 \sim 1.8(10 \mathrm{H}, \mathrm{m}), 2.1 \sim 2.7\left(2 \mathrm{H}, \mathrm{m}, J_{2,3}=9.6 \mathrm{~Hz}\right), 3.0 \sim 3.2(1 \mathrm{H}, \mathrm{br}), 3.6 \sim 4.0(2 \mathrm{H}, \mathrm{m}), 4.3 \sim 4.5(1 \mathrm{H}$, m); yield $1 \%$.

14: ${ }^{1} \mathrm{H}$ NMR $(270 \mathrm{MHz}) \delta 0.8 \sim 0.9(3 \mathrm{H}, \mathrm{t}), 1.2 \sim 2.0(12 \mathrm{H}, \mathrm{m}), 2.3 \sim 2.5\left(2 \mathrm{H}, \mathrm{m}, J_{2,3}=7.0 \mathrm{~Hz}\right)$, $3.6 \sim 3.8(2 \mathrm{H}, \mathrm{m}), 4.0 \sim 4.5(2 \mathrm{H}, \mathrm{m})$; yield $5 \%$.

15: ${ }^{1} \mathrm{H}$ NMR $(270 \mathrm{MHz}) \delta 0.8 \sim 0.9(3 \mathrm{H}, \mathrm{t}), 1.2 \sim 2.0(12 \mathrm{H}, \mathrm{m}), 2.3 \sim 2.5\left(2 \mathrm{H}, \mathrm{m}, J_{2,3}=5.8 \mathrm{~Hz}\right)$, $3.6 \sim 3.8(2 \mathrm{H}, \mathrm{m}), 4.0 \sim 4.5(2 \mathrm{H}, \mathrm{m})$; yield $2 \%$.

\section{Results and Discussion}

\section{Effect of VB-C Analogues Having Various C-2 Side Chains} on Virginiamycin Production

Various VB-C analogues tested for their inducing activity are shown in Fig. 2. They can be divided into three main groups, i.e. group I, analogues having the 3-(hydroxymethyl)butanolide skeleton in common but with various $\mathrm{C}-2$ side chains (compounds $8 \sim 10$ ); group II, analogues with the heptyl moiety at C-2 but lacking a free hydroxy group at C-5 (11 13); group III, analogues lacking the C-6 hydroxy group (14 and 15). Before testing those analogues, we tested butanolide and its 
Fig. 2. Structures of various VB-C analogues.<smiles>[R]C(=O)C1C(=O)OCC1CO</smiles>

8a $\mathrm{R}=\mathrm{CH}_{3}$

$8 b \mathrm{R}=\widehat{ }$

$8 \mathrm{c} R=ح$

8d $\mathrm{R}=ح$

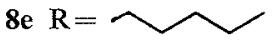

8f $\mathrm{R}=\mathrm{N}^{\mathrm{N}}$

$8 \mathrm{~g}={ }^{8}$

$8 \mathrm{~h} R=ح$

$8 \mathrm{R}=\mathrm{N}^{\mathrm{N}}$

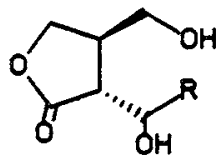

9a $\mathrm{R}=\mathrm{CH}_{3}$

9b $\mathrm{R}=\widehat{ }$

9c $\mathrm{R}=\widetilde{\sim}$

$9 \mathrm{~d} R=\widehat{ح}$

$9 \mathrm{e} \mathrm{R}=\sim$

9f $\mathrm{R}=\mathrm{ح}^{2}$

$9 \mathrm{~g}=\sim$

$9 \mathrm{~h} R=\bigcirc$

$9 \mathrm{i} \mathrm{R}={ }^{2}$

9j $R=ح$

$9 \mathrm{k}=\sim$

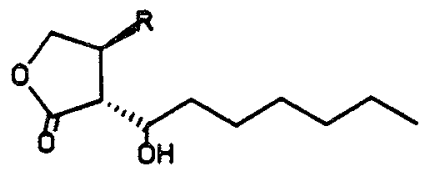

12a $\mathrm{R}=\mathrm{CH}_{2} \mathrm{OCH}_{3}$

12b $\mathrm{R}=\mathrm{CH}_{3}$

12c $\mathrm{R}=\mathrm{H}$<smiles>[R]C(O)[C@H]1C(=O)OC[C@H]1CO</smiles>

10a $\mathrm{R}=\mathrm{CH}_{3}$

10b $\mathrm{R}=ح$

$10 \mathrm{c} R=\Upsilon$

$10 \mathrm{~d} R=ح$

$10 \mathrm{e}=\Upsilon$

$10 \mathrm{~F} \mathrm{R}=ح$

$10 \mathrm{~g}=\frown$

$10 \mathrm{H} \mathrm{R}=$

$10 \mathrm{i} \mathrm{R}=\sim$

10j $\mathrm{R}=\leadsto$

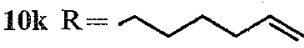

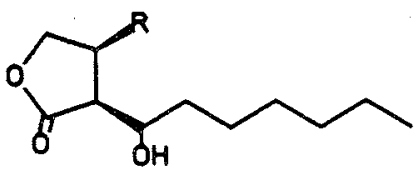

13a $\mathrm{R}=\mathrm{CH}_{2} \mathrm{OCH}_{3}$

13b $\mathrm{R}=\mathrm{CH}_{3}$ 11b $\mathrm{R}=\mathrm{CH}_{3}$

11c $\mathrm{R}=\mathrm{H}$

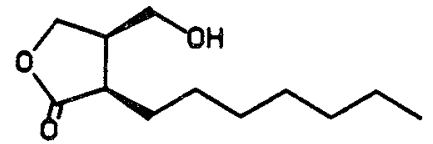

15

analogues (cyclopentane, cyclopentanone, tetrahydrofuran and 2-pyrrolidone) to check the possibility that the butanolide skeleton itself has inducing activity. However, none of them showed inducing activity at $100 \mu \mathrm{g} / \mathrm{ml}$, nor suppressed the inducing activity of $\mathrm{VB}-\mathrm{C}(10 \mathrm{ng} / \mathrm{ml})$ at the concentration of $100 \mu \mathrm{g} / \mathrm{ml}$, indicating that substituents in VB-C are essential in inducing virginiamycin production.

In Fig. 3 is shown an example of the bioassay in which several concentrations of A-factor type analogues (8) were tested. When the concentration of analogues tested was insufficient for virginiamycin production, no clear zone was observed $(8 \mathrm{~mm}$ corresponds to the diameter of the cups). At or above the effective concentration, the diameter of clear zone increased, by which we determined the minimum effective concentration of each analogue (Table 1). By comparing the minimum effective 
Fig. 3. Induction of virginiamycin production by a series of A-factor type analogues.<smiles>[R]C(=O)C1C(=O)OCC1CO</smiles>
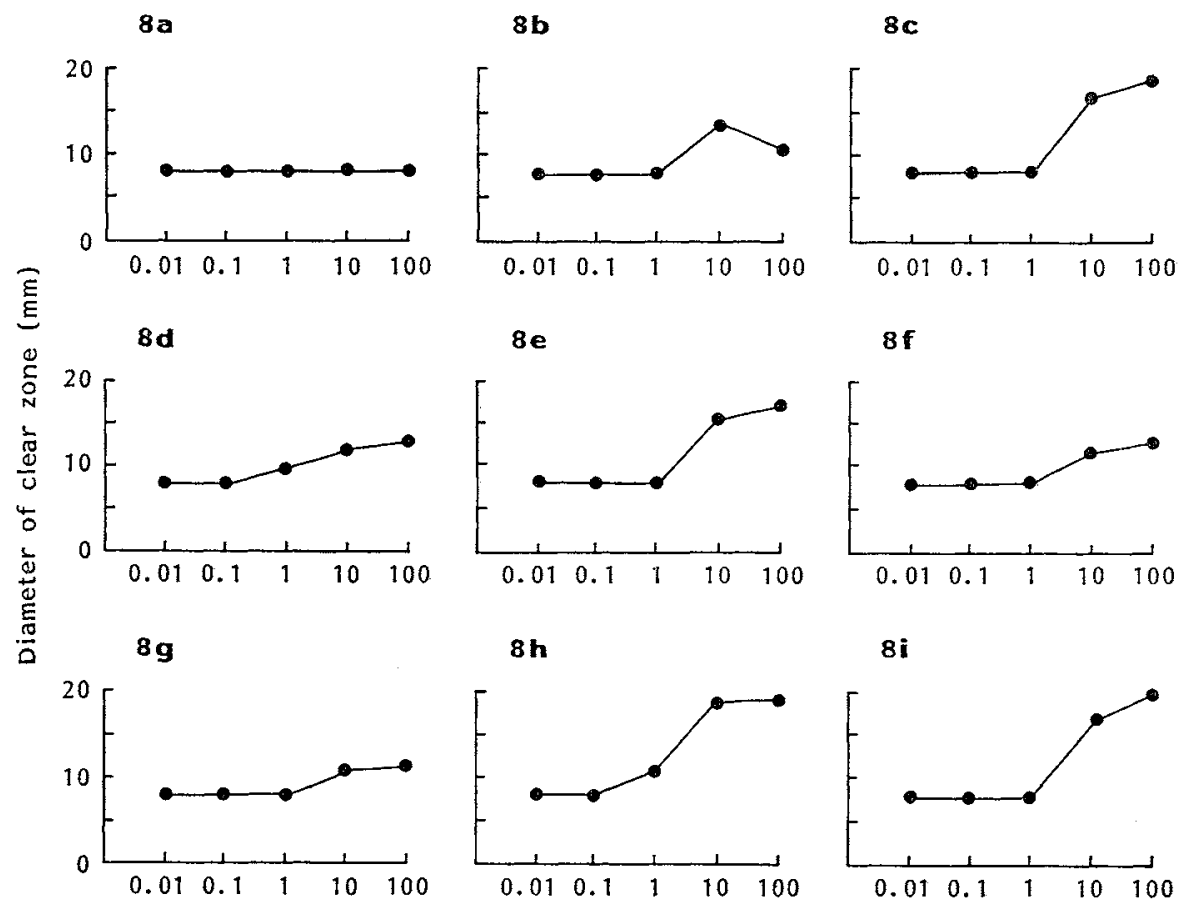

$8 \mathbf{h}$

$8 \mathrm{i}$
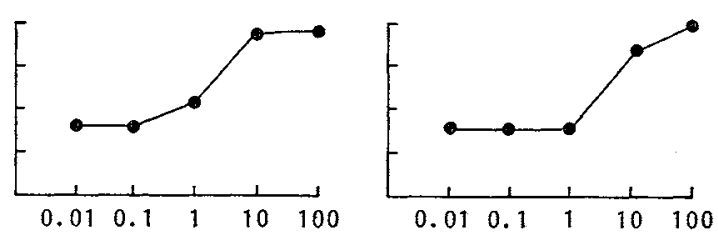

Concentration $(\mu \mathrm{g} / \mathrm{ml})$

Table 1. Minimum effective concentration of VB-C analogues.<smiles>[R]C(=O)C1C(=O)OCC1CO</smiles>

8<smiles>[R]C(O)[C@H]1C(=O)OC[C@H]1CO</smiles>

9<smiles>[R]C(O)[C@H]1C(=O)OC[C@H]1CO</smiles>

10

\begin{tabular}{cccc}
\hline Analogue & $\begin{array}{c}\text { A-Factor type } \\
(\mathbf{8})(\mu \mathrm{g} / \mathrm{ml})\end{array}$ & $\begin{array}{c}\text { trans VB-C type } \\
(\mathbf{9})(\mu \mathrm{g} / \mathrm{ml})\end{array}$ & $\begin{array}{c}\text { cis VB-C type } \\
(\mathbf{1 0})(\mu \mathrm{g} / \mathrm{ml})\end{array}$ \\
\hline $\mathbf{a}$ & $>100$ & $>100$ & 100 \\
$\mathbf{b}$ & 10 & 100 & 100 \\
$\mathbf{c}$ & 10 & 10 & 10 \\
$\mathbf{d}$ & 10 & 1 & 0.1 \\
$\mathbf{e}$ & 10 & 0.1 & 0.003 \\
$\mathbf{f}$ & 10 & 0.01 & 0.0008 \\
$\mathbf{g}$ & 10 & 0.01 & 0.0008 \\
$\mathbf{h}$ & 1 & 0.1 & 0.1 \\
$\mathbf{i}$ & 10 & 1 & 0.1 \\
$\mathbf{j}$ & - & 100 & 10 \\
$\mathbf{k}$ & - & 0.1 & 0.001 \\
\hline
\end{tabular}


concentrations for 8,9 and 10 , it was clear that cis VB-C type analogues (10) were 10 -fold more active than the corresponding trans isomers (9), and 9 were 10 -fold more active than A-factor type analogues (8). Therefore, 2,3-cis configuration is important for inducing activity. This agrees well with the fact that all the natural factors $(3,6$ and 7$)$ from $S$. virginiae have the cis configuration.

Since natural VBs (3,6 and 7) differ only in the substituent at $\mathrm{C}-2$, the moiety at $\mathrm{C}-2$ seems rather flexible. Therefore, to determine the optimum size of the substituent, we synthesized a series of $\mathbf{1 0}$ and compared their effectiveness (Fig. 4). The highest activity was observed with $n$-heptyl or $n$-octyl substituents. These had a minimum effective concentration of $0.8 \mathrm{ng} / \mathrm{ml}$ which was 2.5-fold lower than that for natural VB-C (about $2 \mathrm{ng} / \mathrm{ml}$ ). Longer or shorter sub-
Fig. 4. Effect of length of the side chain at C-2 on the inducing activity of $c i s$ VB-C analogues.
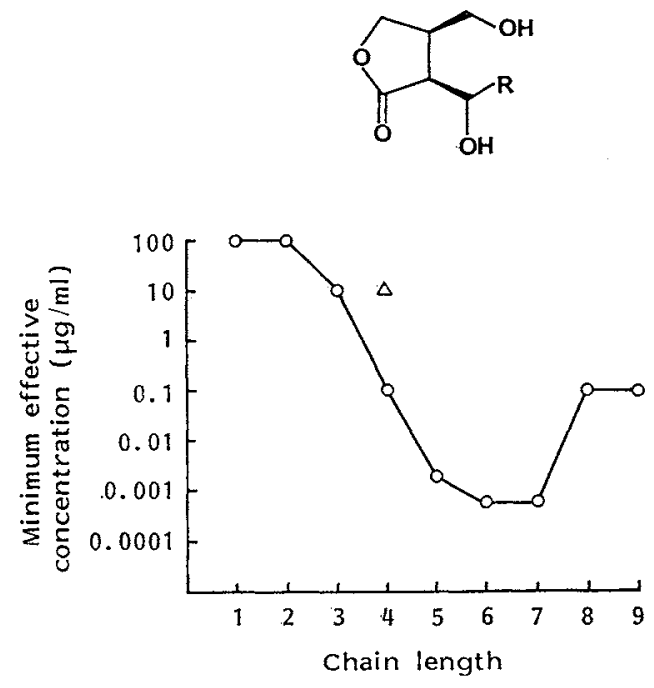

Chain length indicates the number of $n+1$ in $\mathrm{R}=\left(\mathrm{CH}_{2}\right)_{\mathrm{n}} \mathrm{CH}_{3}$. Value for an analogue having a terminal double bond in $R$ is indicated by $(\Delta)$. stituent resulted in lower activity, and the presence of a terminal double bond resulted in a 100-fold decrease of the activity. A similar tendency was observed for the trans type (9) analogues. KAWAGUCHI et al. ${ }^{7)}$ reported that the $n$-octyl ester of B-factor showed the highest activity in the induction of rifamycin production in Nocardia sp. The requirement of an $n$-octyl group for highest inducing activity in different organisms suggests that a hydrophobic interaction with such a non-polar group is important in the process of induction. For A-factor (1) in S. griseus, KHoKHLov ${ }^{2}$ reported that analogues having one carbon atom shorter or longer than the natural A-factor (1) exhibited only $0 \sim 15 \%$ or $0 \sim 10 \%$ activity, respectively. In VB-C, analogues of $n$-heptyl and $n$-octyl showed the same activity. The reason for this difference between A-factor analogues and VB-C analogues is not clear, but may result from the difference between $S$. griseus and $S$. virginiae.

\section{Importance of Hydroxy Groups on the Substituents for Inducing Activity}

By fixing the size of the C-2 side chain to an $n$-heptyl group, we next investigated the importance of the two hydroxy groups on the substituents (Table 2). For the C-5 hydroxy group, methoxylation (13a) caused a dramatic decrease (1,250-fold) in inducing activity. Interestingly, deletion of a free $\mathrm{OH}$ group (13b) or deletion of even a hydroxymethyl group from C-3 (13c) did not cause a further decrease in the activity; they all showed the same minimum effective concentration of $1 \mu \mathrm{g} / \mathrm{ml}$. Therefore, the C-5 carbon atom and oxygen atom do not seem to play an important role in the inducing activity, but the free $\mathrm{OH}$ group seems to be an important group, probably by making a hydrogen bond, although for the oxygen atom in the methoxy analogue (13a), we cannot exclude the possibility that steric hindrance caused by the methyl group was the actual reason for the activity loss.

With regard to the hydroxy group at C-6, its deletion (15) also resulted in a great decrease (125fold) in inducing activity, but less of a decrease than that for the C-5 hydroxy group, indicating that the C-5 hydroxy group is more important than the C-6 hydroxy group in the inducing activity of VB-C. 
Table 2. Minimum effective concentration of VB-C analogues.

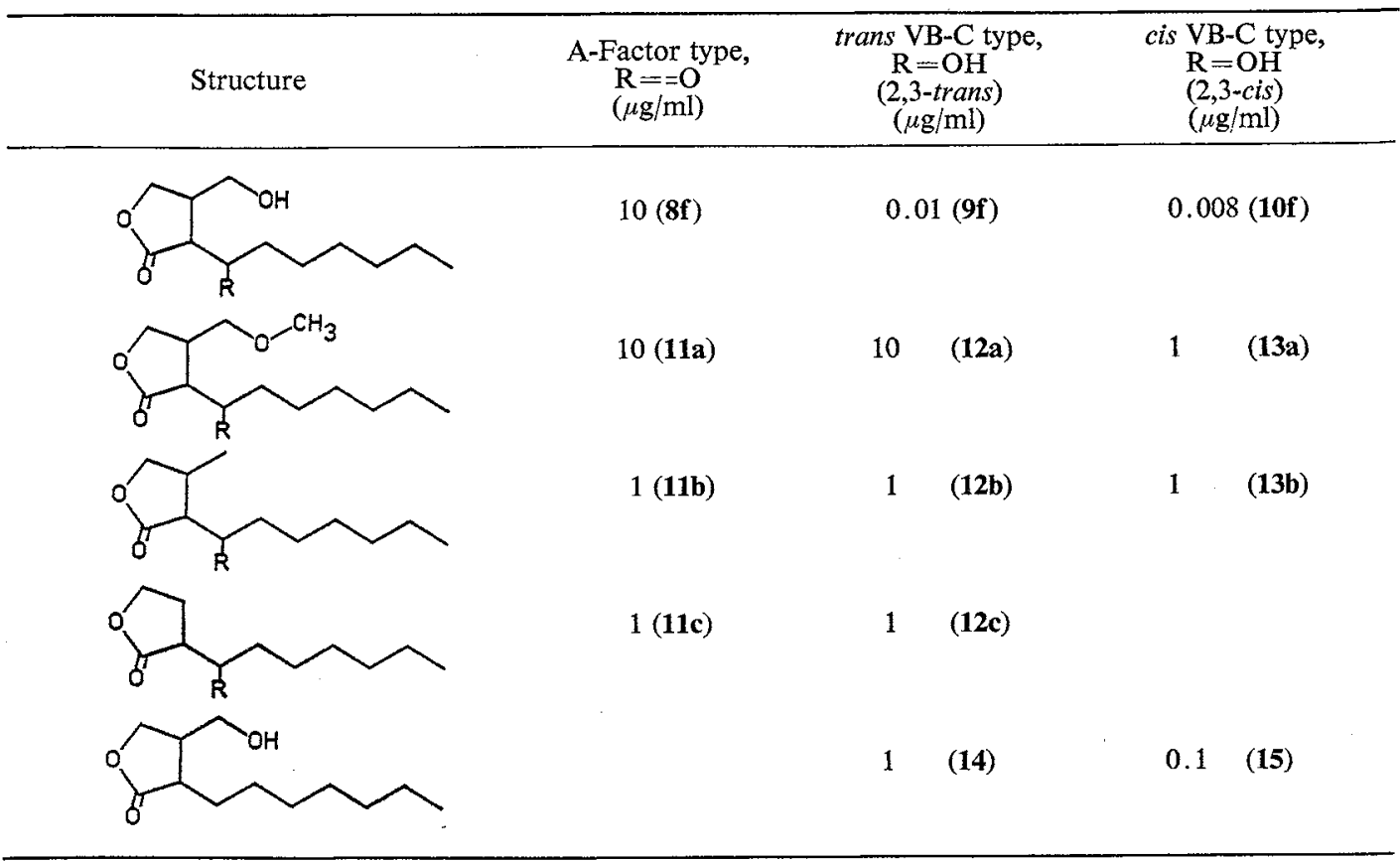

As a conclusion, VB-C can be said to have at least 4 important parts for demonstrating inducing activity, i.e., i) C-5 free hydroxy group, ii) C-6 hydroxy group, iii) 2,3-cis configuration, and iv) C-2 side chain length with an optimum at 7 or 8 carbon atoms. The presence of two oxygen atoms as well as the cyclic structure of the butanolide skeleton may also play important roles in the induction, but these possibilities remain to be determined.

\section{References}

1) Kleiner, E. M.; S. A. Pliner, V. S. Solfer, V. V. Onoprienko, T. A. Balashova, B. V. Rosynov \& A. S. KнокнLOv: The structure of A-factor, a bioregulator from Streptomyces griseus. Bioorg. Khim. 2: $1142 \sim 1147,1976$

2) KHOKHLov, A. S.: Problems of studies of specific cell autoregulators (on the example of substances produced by some actinomycetes). In Frontiers of Bioorganic Chemistry and Molecular Biology. Ed., S. N. Ananchenko, pp. $201 \sim 210$, Pergamon Press, New York, 1980

3) HARA, O. \& T. BEPPU: Mutants blocked in streptomycin production in Streptomyces griseus - The role of A-factor. J. Antibiotics 35: 349 358, 1982

4) GRÄFE, U.; W. SCHADE, I. ERITT, W. F. FLECK \& L. RADICS: A new inducer of anthracycline biosynthesis from Streptomyces viridochromogenes. J. Antibiotics 35: 1722 1723, 1982

5) Gräfe, U.; G. Reinhardt, W. SChade, I. ERitT, W. F. FlecK \& L. Radics: Interspecific inducers of cytodifferentiation and anthracycline biosynthesis from Streptomyces bikiniensis and S. cyaneofuscatus. Biotechnol. Lett. 5: 591 596, 1983

6) Kawaguch, T.; T. Asahi, T. Satoh, T. Uozum \& T. Beppu: B-Factor, an essential regulatory substance inducing the production of rifamycin in a Nocardia sp. J. Antibiotics 37: 1587 1595, 1984

7) Kawaguchi, T.; M. Azuma, S. Horinouchi \& T. Beppu: Effect of B-factor and its analogues on refamycin biosynthesis in Nocardia sp. J. Antibiotics 41:360 365, 1988

8) BIRÓ, S.; I. BÉKÉSI, S. VITÁLIS \& G. SZABó: A substance effecting differentiation in Streptomyces griseus. Eur. J. Biochem. 103: 359 363, 1980

9) Kondo, S.; K. Yasui, M. Katayama, S. Marumo, T. Kondo \& H. Hatrori: Structure of pamamycin607, an aerial mycelium-inducing substance of Streptomyces alboniger. Tetrahedron Lett. 28: 5861 
5864,1987

10) SCribner III, H. E.; T. TANG \& S. G. Bradley: Production of a sporulation pigment by Streptomyces venezuelae. Appl. Microbiol. 25: 873 879, 1973

11) Yamada, Y.; K. Sugamura, K. Kondo, M. Yanagtmoto \& H. OKada: The structure of inducing factors for virginiamycin production in Streptomyces virginiae. J. Antibiotics 40: 496 504, 1987

12) Yanagimoto, M. \& G. Terui: Physiological studies on staphylomycin production. I, on product inhibition. J. Ferment. Technol. 49: 604 610, 1971

13) Yanagimoto, M. \& G. Terui: Physiological studies on staphylomycin production. II, formation of a substance effective in inducing staphylomycin production. J. Ferment. Technol. 49: 611 618, 1971 